Provided for non-commercial research and education use. Not for reproduction, distribution or commercial use.

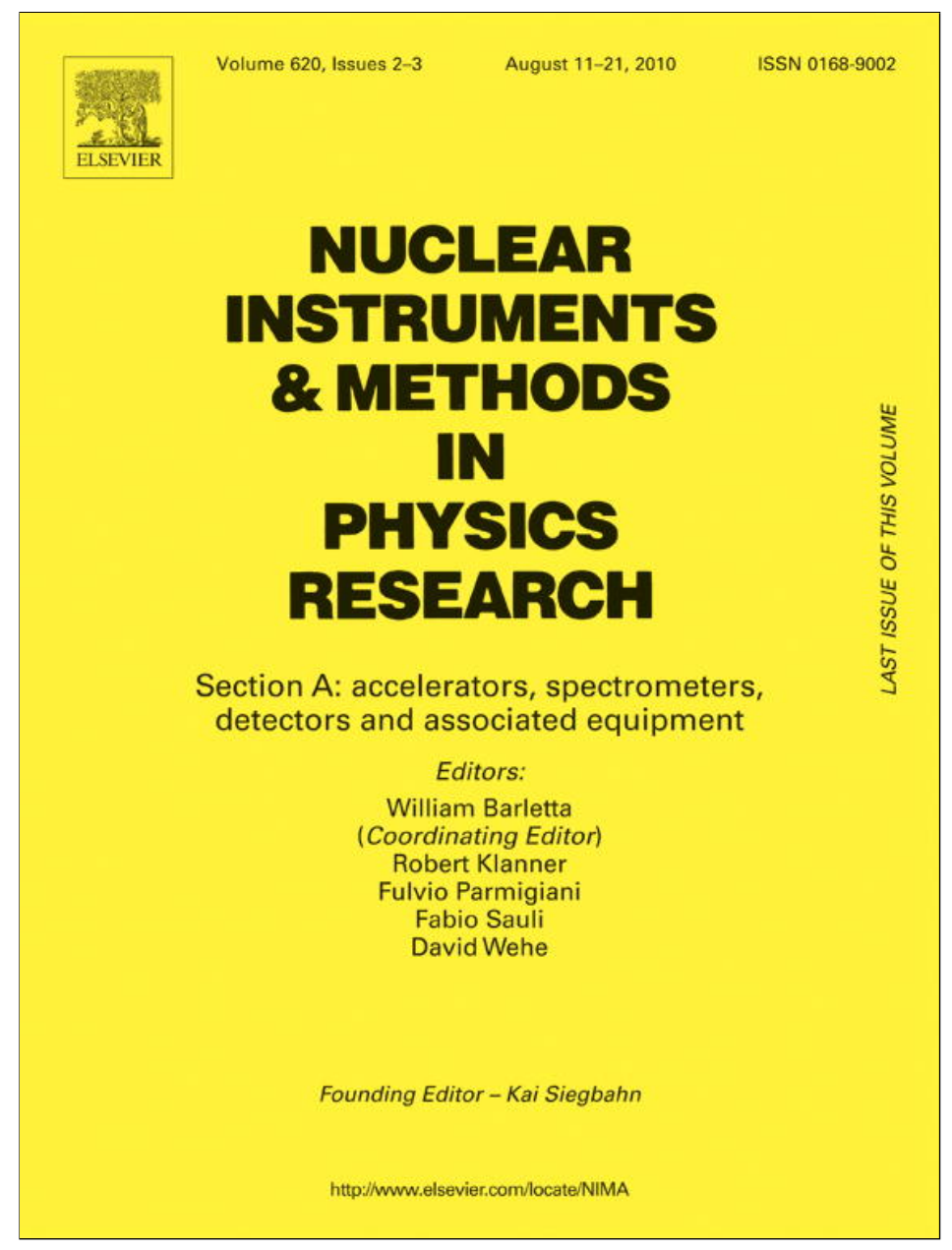

This article appeared in a journal published by Elsevier. The attached copy is furnished to the author for internal non-commercial research and education use, including for instruction at the authors institution and sharing with colleagues.

Other uses, including reproduction and distribution, or selling or licensing copies, or posting to personal, institutional or third party websites are prohibited.

In most cases authors are permitted to post their version of the article (e.g. in Word or Tex form) to their personal website or institutional repository. Authors requiring further information regarding Elsevier's archiving and manuscript policies are encouraged to visit:

http://www.elsevier.com/copyright 


\title{
Apparatus for simultaneous rheology and small-angle neutron scattering from high-viscosity polymer melts and blends
}

\author{
Jitendra Sharma ${ }^{\mathrm{a}, *}$, Stephen M. King ${ }^{\mathrm{b}}$, Leif Bohlin ${ }^{\mathrm{c}}$, Nigel Clarke ${ }^{\mathrm{a}}$ \\ a Department of Chemistry, Science Site Laboratories, University of Durham, South Road, Durham DH1 3LE, United Kingdom \\ b ISIS Facility, Rutherford Appleton Laboratory, Chilton, Didcot, Oxon OX11 0QX, United Kingdom \\ ${ }^{\mathrm{c}}$ Reologen $i$ Lund AB, Öved 19, S-27594 Sjöbo, Sweden
}

\section{A R T I C L E I N F O}

Article history:

Received 31 January 2010

Received in revised form

24 March 2010

Accepted 24 March 2010

Available online 8 April 2010

\section{Keywords:}

Polymer

Melts

Blends

Solutions

Rheometer

Oscillatory and steady shear

Viscosity

Lower critical solution temperature

Neutron and X-ray scattering

\begin{abstract}
A B S T R A C T
In situ study of structural changes in soft matter systems while exposed to shear gives vital information related to the dynamics of polymers. A new shear apparatus has been developed for simultaneous rheology and in situ small-angle neutron scattering (SANS) from high-viscosity polymeric melts and blends. The apparatus described here enables one to perform rheological measurements in a plate-plate geometry under various modes of applied shear viz., steady, oscillatory, and other programmed mode, in a wide range of temperatures that can be varied from ambient to $550 \mathrm{~K}$. A major advantage of this instrument compared to other counterparts (available elsewhere) for a similar geometry of operation is that it is also equipped with a strain sensor for rheological measurements along with the capability to offer both steady state and oscillatory shearing measurements with a single instrument, something hitherto not possible due to the limitations imposed by the conceived design of the instruments. Besides, the parallel plate geometry of the apparatus utilized here offers a distinctive edge over Couette type cells used for similar purposes as the latter is often not suitable for studies on well-entangled concentrated polymeric systems due to high viscosity and associated large sample volume requirements. The details of the design, construction and operation of such device, the Rheo-SANS apparatus, are described in this paper. Preliminary test data obtained from the initial experiments on different samples of blends of deuterated polystyrene and poly(vinyl methyl ether) are presented and discussed within the context of theories known to predict their behaviour.
\end{abstract}

(c) 2010 Elsevier B.V. All rights reserved.

\section{Introduction}

The development of a variety of shear devices over the past few years has facilitated the study of shear-induced structural transformations in soft condensed matter systems in situ using small-angle scattering experiments with X-rays (SAXS) [1], light (SALS) [2], and neutrons (SANS) [3]. The ability of neutrons to penetrate through the complex geometry of shear apparatus along with the contrast achieved from the deuterium labelling method offers SANS a distinctive edge over other radiation scattering techniques. Thus, small-angle neutron scattering (SANS) has proven to be one of the most powerful techniques to probe in situ structural changes in complex fluids under shear. The availability of new devices capable of being operated in situ during scattering measurements has enabled scientists to get new insights into the underlying physics of various polymeric systems under flow. Also, our experiments [4] clearly underline the

\footnotetext{
* Corresponding author. Present address: School of Physics, Shri Mata Vaishno Devi University, Katra 182320, J\&K, India.

E-mail address: jitendra.sharma@gmail.com (J. Sharma).
}

synergistic link between rheology and thermodynamics in polymeric blend systems and the need for simultaneous study of the transient structures (SANS) during the shear. Beginning with the first report on shear-induced effects on the behaviour of colloidal crystals [5], the field of polymer science has seen an explosion in the studies of such non-equilibrium phenomenon where flow induces structural transformations over length scales, extending from nanometers to several microns. These include, for example, shear-induced deformation of polymer coils in dilute and semi-dilute solutions [6], shear-induced orientation of the body-centred-cubic phase in a diblock copolymer gel [7], shear alignment of rod-like polymer micelles [8], a shear-induced firstorder isotropic to nematic phase transition [9], shear-induced transition of lamellar to multilamellar vesicle formation [10], and shear-induced mixing and de-mixing in polymer blends $[11,12]$. Of particular interest is the effect of the rate of shear on the structure and orientation of multi-component systems. For example, a shear-induced nano-macro structural transition in the fluid structure, i.e., low shear rate flow-induced nanometerscale anisotropy to high shear rate bulk phase separation on the micron-scale, has been observed for bicontinuous microemulsions [13]. These studies led to the design and construction and 
installation of many different shear devices at several neutron scattering facilities all over the world. Scientists and engineers have tried relentlessly to improve the performance and efficiency of many such devices to cope with the increasing demand of the experimental requirements largely dictated by the viscosity of the sample, observation geometry, and the mode of applied stress. Therefore, the phase for the development of shear apparatus suitable for in situ measurements has passed through several stages incorporating relevant changes and modifications to fulfil these demands.

To date, SANS from systems under flow has primarily been performed with Couette geometry cells, primarily because the systems under study are low viscosity fluids. The main use of SANS experiments on sheared systems is to align anisometric particles and micelles for the purpose of overcoming Brownian orientational averaging $[14,15]$ or to re-orient mesophases [16] and induce structural changes in them $[17,18]$. In polymer solutions, SANS has been used to study the shear dependence of both single chain conformations in dilute and semi-dilute polymer solutions [6] and concentration fluctuations in semidilute polymer solutions [19]. However, Couette cells are unsuitable for concentrated well-entangled solutions melts and blends, due to their high viscosity and the associated large sample volume requirements. For such systems plate and plate, or cone and plate geometries are preferable for the study of rheological properties and so far only a limited number of SANS experiments have utilized such cells $[3,20]$. However, even these instruments are unable to perform all the desired operations for Rheo-SANS. While the instrument designed by Kalus et al. [3] can be used to perform in situ SANS measurements in those geometries, it does not contain the requisite strain sensor to obtain simultaneous rheology. Though another apparatus designed by Mortensen et al. [20] performs simultaneous rheology, it is limited only to oscillatory shear measurements and simply cannot perform steady shear measurements because of the limitations imposed by the design of the instrument. The new apparatus described here overcomes these shortcomings and includes both the capabilities in a single piece of apparatus. Some comments regarding similar Rheo-SAXS instrument are pertinent here. And, therefore, it must be emphasized that SANS and SAXS, whilst being complementary techniques, generally probe different behaviour; for example, due to isotopic labelling, only SANS can provide information regarding single chain structure factors and is sensitive enough to measure concentration fluctuations. It is less challenging to design Rheo-SAXS cells because the beam is far smaller, but such designs do not translate easily to a Rheo-SANS instrument like the one described here. The details of the design, construction and operation of such a shear device for Rheo-SANS measurements, which enables simultaneous study of the mechanical response and the associated change in the structural properties of polymer melts and blends in a plate-plate geometry under various modes of applied shear, are presented here.

This paper is organised as follows. Section 2 summarizes the details of design, construction and operational features of the Rheo-SANS apparatus. Section 3 outlines the procedure and the materials and methods used in the experiment. In Section 4, we present the details of the results and discussion of the experimental observations obtained from preliminary experiments. Finally, Section 5 concludes the summary and shortcomings while presenting the scope for possible improvements in future.

\section{Apparatus}

Fig. 1 shows an overall view of the Rheo-SANS apparatus. This is a controlled-strain plate-plate rheometer, but it is in horizontal

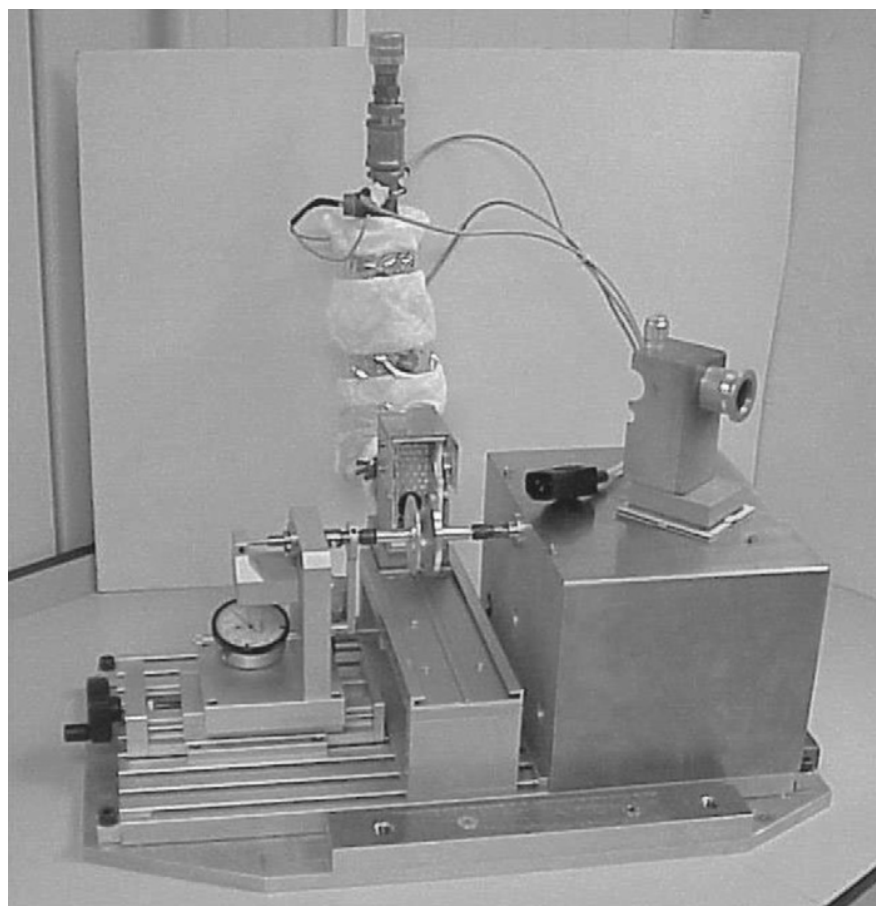

Fig. 1. A pictorial view of the Rheo-SANS apparatus fitted on its beam line mounting plate. For a clearer picture of all the internal components, one part of the "clamshell" oven has been removed temporarily and placed on top of the motor box.

geometry (i.e. the plates are vertical) to suit the requirements of the SANS beam line. The rheometer is controlled by an electronics box, which in turn is interfaced with a computer/laptop for issuing commands. The electronics communicates via a serial port with the computer/laptop and is driven by an adaptation of the state-of-the-art user-friendly VOR Millennium ${ }^{\mathrm{TM}}$ software running under Microsoft Windows ${ }^{\mathrm{TM}}$. The electronics unit houses the torque, amplitude and the servo boards with a dedicated microprocessor to handle the control and measurement functions with VOR Millennium ${ }^{\mathrm{TM}}$. Details of the design, construction and operational features of the Rheo-SANS apparatus are described in the following subsections.

\subsection{Rheometer}

An initial design was drawn in conjunction with Reologen i Lund $A B$ (Sweden) and has been subsequently modified to suit the geometrical constraints of the LOQ diffractometer at the ISIS facility, Rutherford Appleton Laboratory. The main features of the Rheo-SANS apparatus are shown in Fig. 2. The coordinate systems representing the direction of flow $(v)$, velocity gradient $(\nabla v)$, and vorticity $(z)$ are shown on top of the diagram. The overall dimension of the instrument on the optical bench base-plate (1) is $500 \mathrm{~mm}(L) \times 250 \mathrm{~mm}(W) \times 246 \mathrm{~mm}(H)$. The rheometer base (1), linear stage (14) mounted on a dovetail slider (15), and the base (12) of the heating chamber are all made of aluminium. Two detachable circular parallel disks ( 7 and 8 ) machined from aluminium alloys for neutron transparency are housed inside the heating chamber (9), which consists of a two-part "clamshell" oven held together by the coupling clamp (10). Fig. 3 shows the view of parallel plates when they are separated (but close to each other). In fact, the plate (7) attached to the motor is of slightly larger diameter (exceeds the other parallel plate by approximately $0.5 \mathrm{~m}$ ) and slightly dished (machined into the shape of a petri-dish of height $\sim 1.0 \mathrm{~mm}$ ) so that the other plate 


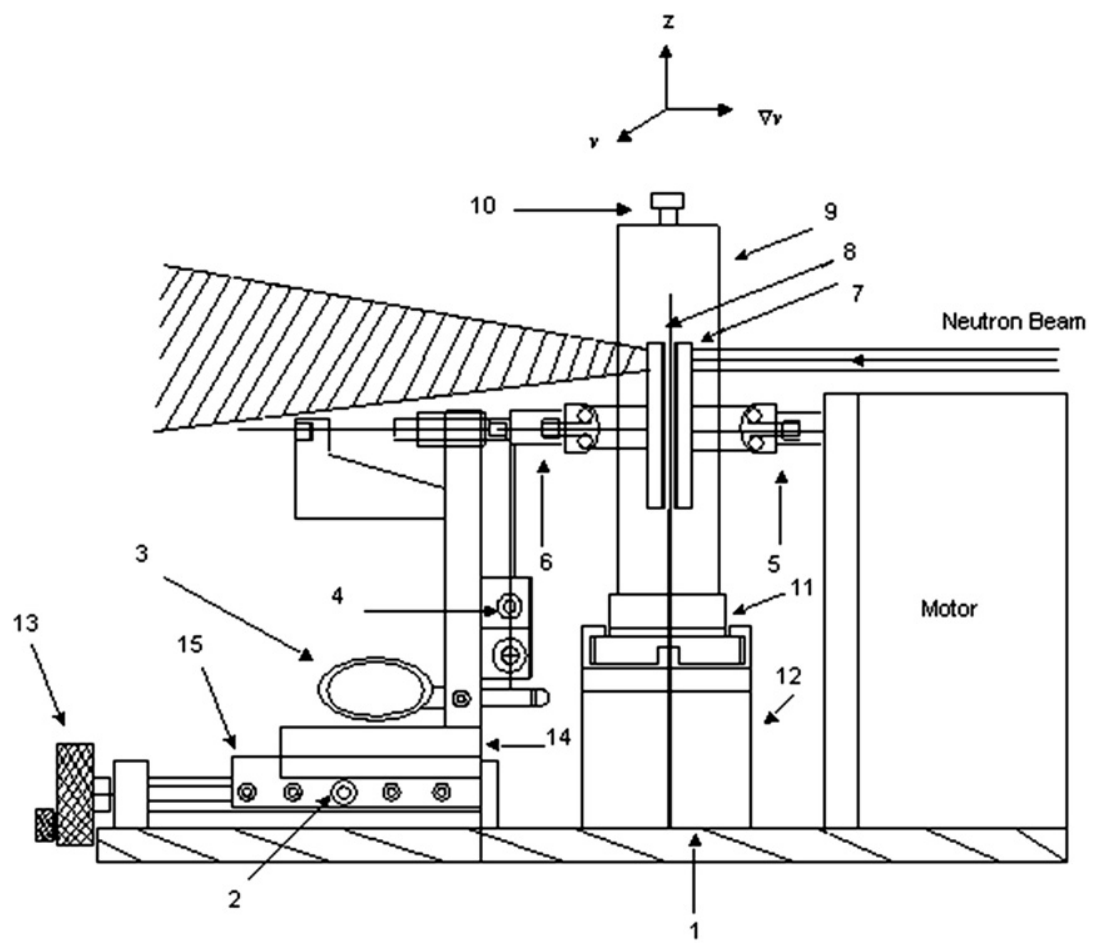

Fig. 2. Schematic view of the Rheometer. The meaning of the numbers, the parts associated with them and their functions are explained in the text. Direction of flow $(v)$, velocity gradient $(\nabla v)$, and vorticity $(z)$ are shown in the co-ordinates drawn on top of the diagram.

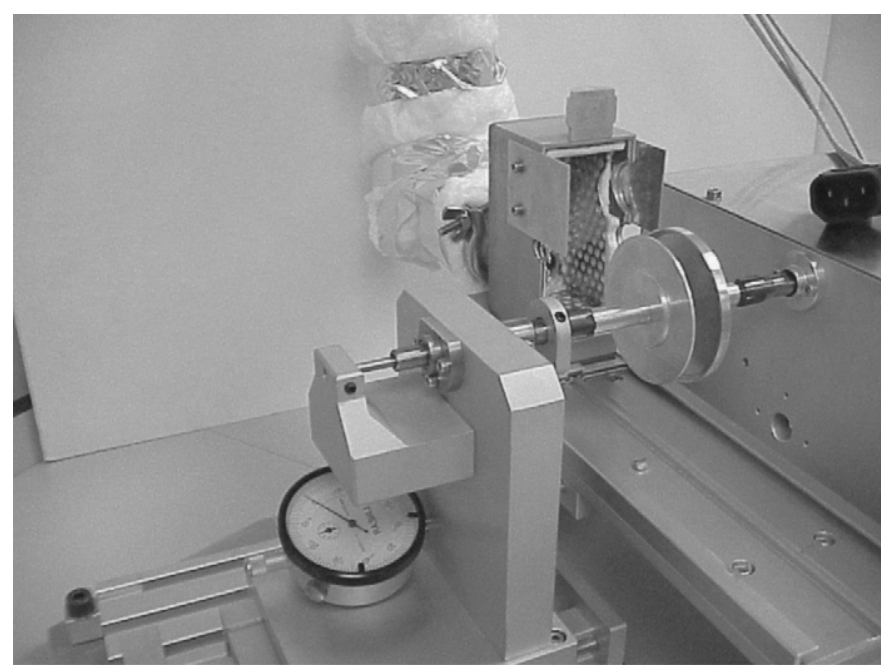

Fig. 3. A close view of the parallel plates when they stand separated but close to each other (there is no sample in between).

(8) may just fit inside. This prevents fluid sample from leaking out and thus provides ideal sealing for high-viscosity polymer melts and blends. During the experiment, these two parallel plates each of thickness 1.5 and $60 \mathrm{~mm}$ in diameter are held together with the sample sandwiched between them. The plate spacing between them can be varied from contact to several centimeters, but is normally set to be $1-3 \mathrm{~mm}$ for SANS measurements. The rotating plate (7) mounted on the actuator (5) via spindle is driven by a motor and applies the commanded value of the strain/stress in the continuous, sinusoidal, transient or a programmed mode. The second plate ( 8 ) is connected to the torque transducer assembly (6) by an attached shaft. This plate (8) along with the torque transducer assembly (6) is mounted on a support, which can be shifted by means of another linear stage (14) mounted on a dovetail slider (15). This linear stage (14) is fitted with a micrometer gauge (13) that allows adjustment of the gap between the parallel plates within a precision scale of $0.01 \mathrm{~m}$ as indicated by the dial indicator (3).

The versatility of the apparatus offers various operational modes that allows measurements in steady shear or viscometry, oscillatory, strain sweep, stress relaxation and transient or a programmed mode of operation. While the viscometry mode allows data collection in steps of various steady shear rates, the oscillatory mode is used for measurement during small-amplitude oscillations. Oscillatory measurements at a fixed frequency can also be carried out during the step increase/decrease of strain using strain sweep mode. Similarly, stress relaxation is used for small-amplitude relaxation experiments while a programmed run allows for the automatic run of a sequence of predefined parameter files in various operational modes. The actuator controlling the torque allowed the rate of shear to vary across four orders of magnitude (between $\Gamma=10^{-3}$ and $10 \mathrm{~s}^{-1}$ ) while running in the rate sweep mode and a frequency-range of $0.001-20 \mathrm{~Hz}$ for an oscillatory run. A variable sensitivity of the torque transducer allows measurements to be performed on samples of low to high viscosities.

\subsection{Temperature control and the heating unit}

Fig. 4 shows a schematic of the oven that houses the parallel plates. This has a "clamshell" design to allow access to the plates for sample changing. To show them separately and a clear inside view of the clamshell oven, one of the detached parts has been removed temporarily (sitting on top of the motor box) in Fig. 1 while the other remains seated on the dovetail base. The oven is manufactured in mild steel but because this would interfere with the SANS measurements, $25 \mathrm{~mm}$ diameter "beam windows" have been machined away on either side across the joint between the two halves of the oven. These "windows" have then been faced with $1 \mathrm{~mm}$ thick aluminium plate. Inside the oven, and except for a cut-out to match the "beam windows", is a lining of $0.25 \mathrm{~mm}$ 


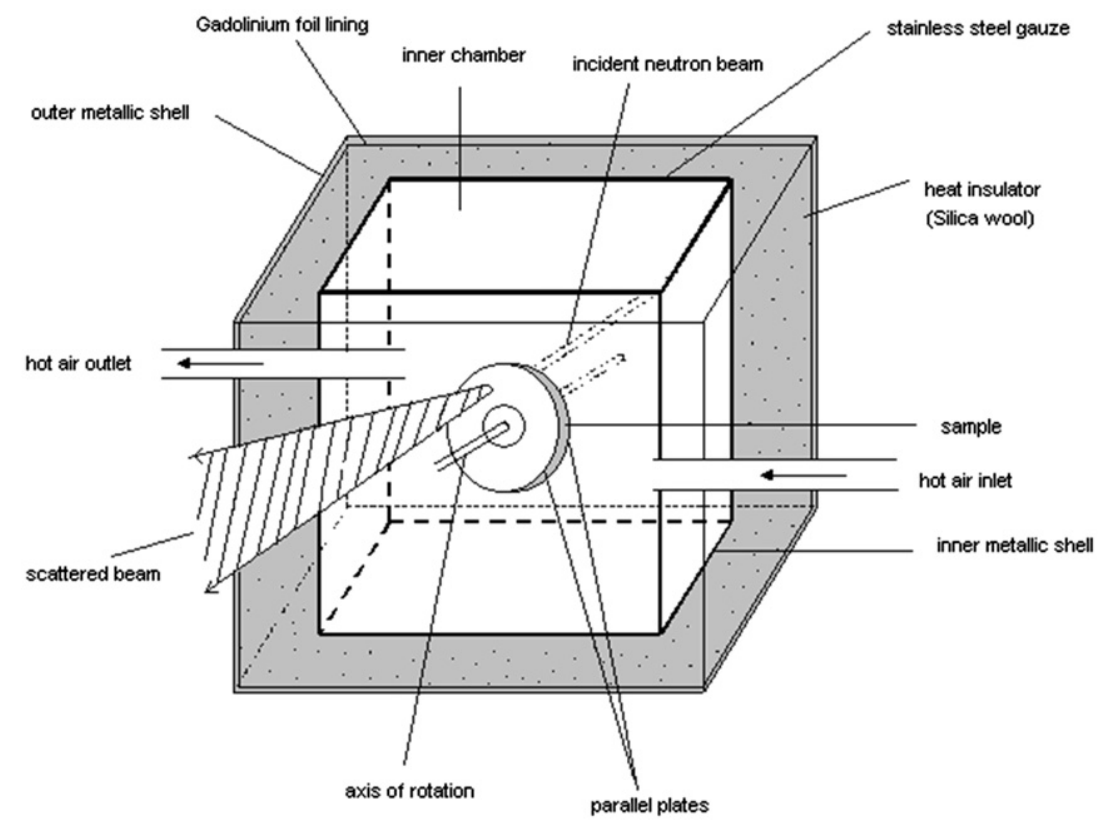

Fig. 4. Cross-section of the heating chamber.

thick gadolinium foil (a potent neutron absorber) and $5 \mathrm{~mm}$ thick silica wool (for insulation), held in place by stainless steel gauze. Each half of the oven is fitted with a standard quick-release vacuum coupling. If necessary this permits the oven to be dynamically pumped to reduce the oxidation of a sample, but also serves to allow the fitment of ancillary equipment such as a heater or air/inert/cryogenic gas purge. The heater used is a $750 \mathrm{~W} 240 \mathrm{~V}$ AC air process heater (RS Components, Model 200-2531), regulated by a K-type thermocouple in the gas stream on the other half of the oven and is connected to a temperature controller (Eurotherm, Model 2404). When connected to a laboratory compressed air service this arrangement provides temperature control over the range from ambient to $550 \mathrm{~K}$.

\section{Experiment}

\subsection{Materials}

Polymer blends of poly(vinyl methyl ether) (PVME) with different molecular weights of deuterated polystyrene (PSD) in varying compositions were studied using the Rheo-SANS apparatus. Samples of polydisperse $\left(M_{\mathrm{w}} / M_{\mathrm{n}}=1.95\right)$ PVME having an approximate molecular weight of 90000 were obtained from Scientific Polymer Products Inc. Different versions of the deuterated polystyrene used for the blend preparations were obtained from two different sources: three low molecular weight deuterated polystyrene of molecular weights $20 \mathrm{~K}\left(M_{\mathrm{w}}=22,770\right), 40 \mathrm{~K}$ $\left(M_{\mathrm{w}}=40,491\right)$, and $300 \mathrm{~K}\left(M_{\mathrm{w}}=307,900\right)$ were synthesized in the laboratory using the standard technique of anionic polymerisation of styrene- $d_{8}$ in benzene with sec-butyllithium as an initiator [21], while the other having a higher molecular weight of $500 \mathrm{~K}$ $\left(M_{\mathrm{w}}=507,695\right)$ was purchased from Polymer Laboratories. All the four PSDs had narrow molecular weight distributions of 1.03 , $1.04,1.04$ and 1.06 , respectively. The blends prepared from 20 and $40 \mathrm{~K}$ deuterated polystyrenes were of 30:70 (\% wt/wt) compositions and the ones from 300 and $500 \mathrm{~K}$ were 50:50 (\% wt/wt), respectively. The blends were prepared from a ternary solution of PSD, PVME and toluene (solvent) and cast into a $60 \mathrm{~mm}$ diameter PTFE mould. The solvent was removed by drying the blends in a vacuum oven for several weeks at $70{ }^{\circ} \mathrm{C}$. The thickness of the plaques was chosen to be $\sim 1 \mathrm{~mm}$, to avoid the effects of multiple scattering. These solid plaques were placed between the parallel plates for the experiments where they were then heated.

\subsection{Rheo-SANS: rheology and small-angle neutron scattering}

A simultaneous study of the mechanical response and the change in the structure of the blends subject to applied stress was carried out using the Rheo-SANS apparatus installed on the LOQ diffractometer [22] at the ISIS pulsed neutron source, Rutherford Appleton Laboratory [23], a white beam instrument using timeof-flight techniques. A wavelength range of 2.2-10.0 $\AA$ at a frequency of $25 \mathrm{~Hz}$ gave a range of scattering vector $q$ (given by $q=(4 \pi / \lambda) \sin (\theta / 2)$, where $\theta$ is the scattering angle and $\lambda$ is the wavelength) of $0.009-0.28 \AA^{-1}$. The scattered neutrons were detected by a two-dimensional ${ }^{3} \mathrm{He}-\mathrm{CF}_{4}$ filled area detector with an active region of $64 \mathrm{~cm} \times 64 \mathrm{~cm}$ with $5 \mathrm{~mm}$ spatial resolution. The typical data-collection time for each run was around 40$50 \mathrm{~min}$. The scattering data were corrected and normalized using standard procedures [24]. The Rheo-SANS apparatus was installed such that the beam hits the parallel plates vertically above the axis of rotation. The shear gradient, which is parallel to the axis of rotation, is then given by $\Gamma=(\omega r) / d$, where $\omega$ is the angular velocity, $d$ is the distance of separation between the plates and $r$ is the distance from the axis of rotation. Since the diameter of the incident neutron beam was only $6 \mathrm{~mm}$, the change in the velocity gradient over the illuminated region of the sample is negligible.

Oscillatory shear rheology was performed at small amplitude of applied strain $(\gamma=5 \%)$, mainly to ensure that the measurements fell in the linear regime of the blends' viscoelastic response behaviour. Isothermal frequency-sweep curves of the storage $\left(G^{\prime}\right)$ and the loss $\left(G^{\prime \prime}\right)$ modulus were obtained from the blends in the homogenous regime of the phase diagram.

\section{Results and discussion}

Recent studies of the thermo-mechanical response obtained from the blends of polystyrene (PS) and PVME near the critical 
region suggest a thermo-rheologically complex behaviour [25], leading to the breakdown of the empirical time-temperature superposition (tTS) principle [26]. Such a complexity is apparently caused by the huge difference in the glass-transition temperature of the constituent components. Different phase behaviours for the blends of PS/PVME have been reported near the critical region under conditions of quiescent (zero shear) and applied shear. While a mean-field to Ising transition of the concentration fluctuations, close to the critical temperature, has been observed under quiescent conditions [27], interesting phenomena of shearinduced mixing and de-mixing occurred when flow fields were applied [11,12]. However, rheological data from almost all experiments carried out in the homogenous but far from the critical region have all exhibited a universal William-LandelFerry (WLF) behaviour [26]. Oscillatory shear experiments on the PSD/PVME blends have been carried out to test whether the structure-property relation obtained from the simultaneous SANS and rheological measurements comply with the predicted WLF behaviour or not while the system is still in the homogenous but far from the critical region.

In the light of the fact that blends of PSD/PVME fall in the category of binary systems exhibiting lower critical solution temperature (LCST) behaviour [28], SANS and oscillatory shear rheology were carried out simultaneously on each blend in steps of $10^{\circ} \mathrm{C}$ (far from the critical temperature) and $5{ }^{\circ} \mathrm{C}$ (approaching the phase boundary) increase in the temperature as the system approaches the lower critical solution temperature. SANS data were recorded first at each temperature and a frequency-sweep in order to obtain storage $\left(G^{\prime}\right)$ and loss $\left(G^{\prime \prime}\right)$ modulus was performed. Steady shear measurements were performed on the low viscosity samples of 30:70 PSD-20 K/PVME-90 K and PSD-40/PVME-90 K between shear rates $1.84 \times 10^{-3}$ and $4.62 \times 10^{-3} \mathrm{~s}^{-1}$.

\subsection{SANS}

SANS has been widely used to interpret the miscibility of binary polymer mixtures using the de Gennes random phase approximation (RPA) approach [29]

$S^{-1}(q)=S_{A}^{-1}(q)+S_{B}^{-1}(q)-2 \chi$

where $S(q)$ is the total scattering structure factor containing information on both the intermolecular and intramolecular correlations between polymer segments and $S_{i}(q)$ is the singlechain structure factor describing the intramolecular correlations of the polymers $(i=\mathrm{A}, \mathrm{B})$ at the scattering vector $q$ and $\chi$ is the Flory-Huggins interaction parameter. Inserting the relevant parameters describing the constituent macromolecules in the blend, Eq. can be re-written as

$K_{N} / S(q)=\left[\phi_{A} N_{A} v_{A} S_{D}\left(q R_{g, A}\right)\right]^{-1}+\left[\phi_{B} N_{B} v_{B} S_{D}\left(q R_{g, B}\right)\right]^{-1}-2 \chi / v_{0}$

where $\phi_{i}, N_{i}$ and $v_{i}$ are the volume fraction, degree of polymerisation and monomer molar volumes of the component $i$, respectively, and $v_{0}$ is the molar volume of the reference cell. $K_{N}$ defines the contrast factor and $S_{D}\left(q R_{g, i}\right)=\left(2 / x^{2}\right)\left(e^{-x}+x-1\right)$ is the Debye scattering function for the Gaussian coils with $x=q^{2} R_{g, i}^{2}$ and $R_{g, i}$ is the radius of gyration. In the limit of small $q$ vectors, Eq. (2) is approximated by the Ornstein-Zernike $(\mathrm{O}-\mathrm{Z})$ behaviour

$S(q)=S(q=0)\left(1+q^{2} \xi^{2}\right)^{-1}$

where $\xi$ is defined as the correlation length. As the spinodal is approached, the correlation length and the zero wave vector structure factor, diverge as

$\xi \sim\left(\chi_{s}-\chi\right)^{-1 / 2}$

$S(0) \sim\left(\chi_{s}-\chi\right)^{-1}$

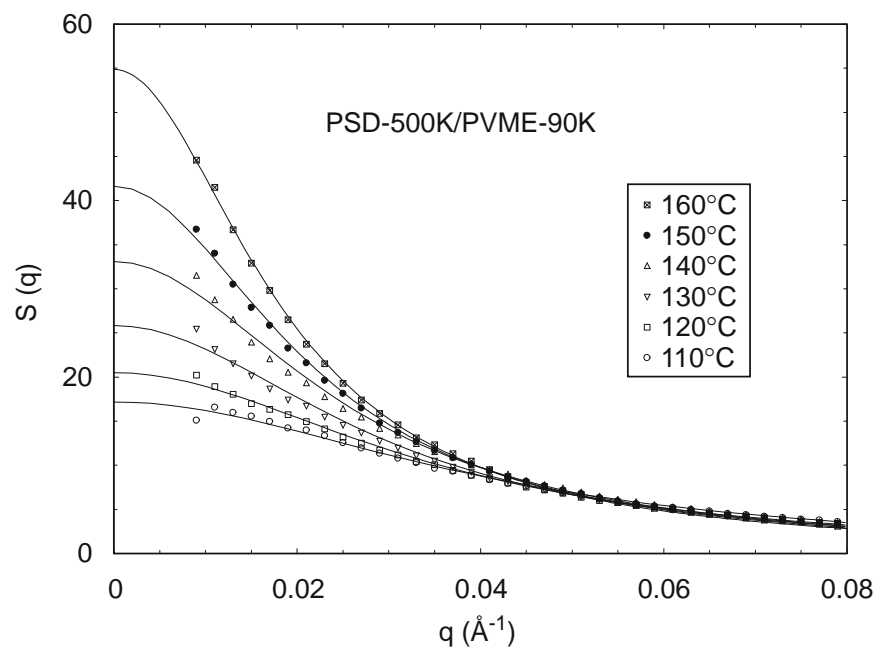

Fig. 5. Plot of the scattering profile as a function of temperature for the 50:50 PSD$500 \mathrm{~K} / \mathrm{PVME}-90 \mathrm{~K}$ blend. Solid lines in the figure represent fits to the $\mathrm{O}-\mathrm{Z}$ function. The error bars have been removed from the data points for clarity.

where $\chi_{s}$ defines the value of $\chi$ at the spinodal. ${ }^{\text {th }}$ Assuming an inverse temperature dependence of the interaction parameter $(\chi \sim a+b / T$ where $a$ and $b$ are constants), the spinodal temperature can be estimated from the intercept of the straight line with the $x$ axis from the plot of $\xi^{-2}$ versus $1 / T$ or $S^{-1}(0)$ versus $1 / T$.

Fig. 5 shows typical neutron scattering data from a 50:50 blend of PSD-500 K/PVME-90 K at six different temperatures. The solid lines in the figure represent fits to the $\mathrm{O}-\mathrm{Z}$ function defined in Eq. (3). The figure clearly shows evidence of strong scattering in the low $q$ region as the system approaches the critical region. A more precise estimation of the zero wave vector scattered intensity is possible by plotting $S^{-1}(q)$ versus $q^{2}$ in the low $q$ region and extract the estimated value of $S^{-1}(0)$ from the extrapolated intercept (with the $y$-axis) of the straight line fit to the data. The ratio of slope/intercept obtained from the fit yields the value of square of the correlation length, $\xi^{2}$ (Eq. (3)). Eq. (4) can now be used to estimate the spinodal temperature of the blend from the intercept (with the $x$-axis) of the straight line fit to the $\xi^{-2}$ versus 1/T. Fig. 6 shows such fits to estimate the spinodal temperature for two different polymer blends of PSD and PVME having composition of $50: 50$ with the molecular weight ratios of $300 \mathrm{~K} / 90 \mathrm{~K}$ and $500 \mathrm{~K} / 90 \mathrm{~K}$. The spinodal temperature thus estimated for the blends had values of $\sim 158$ and $\sim 173{ }^{\circ} \mathrm{C}$ for the former and latter, respectively.

\subsection{Rheology}

\subsubsection{Steady shear}

Steady shear measurements were performed on the samples of low viscosity blends in the homogenous state. The shear rates available for 30:70 compositions of PSD-20 K/PVME-90 K and PSD-40 K/PVME-90 K for the temperatures studied were between $1.84 \times 10^{-3}$ and $4.62 \times 10^{-3} \mathrm{~s}^{-1}$. Simultaneous SANS and steady shear viscosity data were recorded at $10{ }^{\circ} \mathrm{C}$ increment for each sample. Fig. 7 shows a typical SANS profile obtained from the blend of PSD-40 K/PVME-90 $\mathrm{K}$ at $90{ }^{\circ} \mathrm{C}$ for different shear rates. A jump in the scattered intensity is clearly observed (compared to the quiescent profile) at the introduction of shear, which continues further as the rate of shear is increased. However, with further increase in the shear rate, the magnitude of the increase in the scattered intensity decreases systematically although the system viscosity decreases continuously (as shown in the inset of Fig. 7). In certain cases (not shown here), such an 
increase has been found to saturate at higher rates of shear between $2.91 \times 10^{-3}$ and $3.67 \times 10^{-3} \mathrm{~s}^{-1}$. Such a phenomenon has been observed previously using light scattering from blends of EVA/SCPE [11]. At a fixed temperature, an increase in scattering was found at low shear rates, indicating an enhancement of phase separation in the two phase region, but at higher shear rates the behaviour was reversed with shear apparently suppressing phase separation. As will be discussed in a future paper, the advantage of SANS over light scattering is that the scattering intensity is more sensitive to small changes in molecular behaviour.

\subsubsection{Oscillatory shear}

The viscoelastic response of the blends were recorded at various temperatures in the SANS-determined homogenous region (far from the critical temperature) of phase diagram. Only the data obtained from the isothermal frequency-sweep curves of the storage $\left(G^{\prime}\right)$ and loss $\left(G^{\prime \prime}\right)$ modulus are presented and analysed

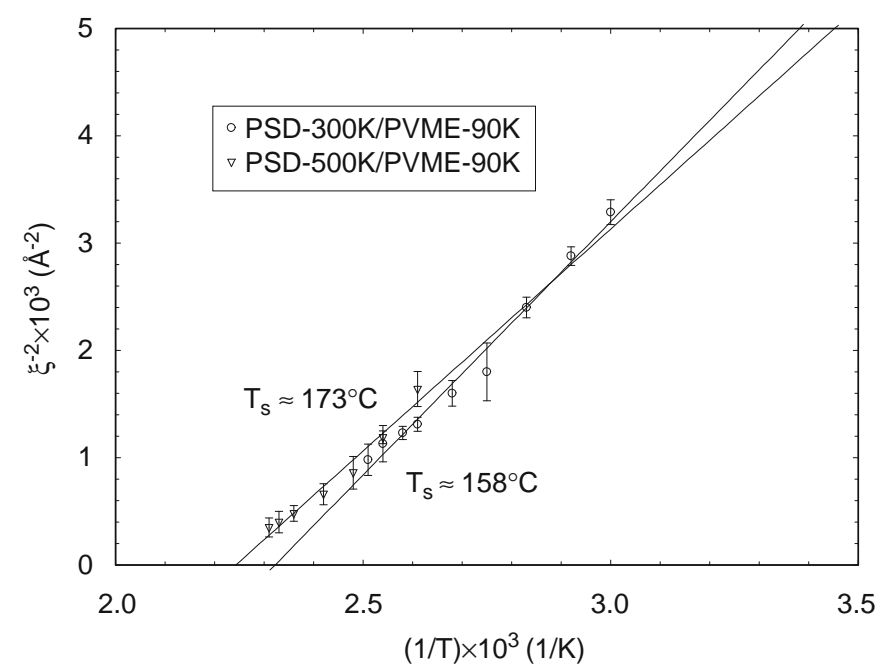

Fig. 6. Estimation of the spinodal temperature from plots of $\xi^{-2}$ versus $1 / T$ for the blends of PSD-300 K/PVME-90 K and PSD-500 K/PVME-90 K. The estimated values of the spinodal temperatures are $\sim 158$ and $\sim 173{ }^{\circ} \mathrm{C}$, respectively. within the purview of the viscoelastic response theory developed for polymer melts and blends. Based on the time-temperature superposition (tTS) principle [26], the frequency $(\omega)$ dependence of the complex modulus $\left(G^{*}\right)$ at any temperature $T$ can be determined from a master curve obtained at a reference temperature $T_{\text {ref }}$ using the equation

$G^{*}(\omega ; T)=b_{T} G^{*}\left(a_{T} \omega ; T_{r e f}\right)$

where $a_{T}$ and $b_{T}$ are the required horizontal (frequency-scale) and vertical (modulus-scale) shift factors, respectively, to allow the superposition of the viscoelastic data at temperature $T$ with the data at the reference temperature. The William-Landel-Ferry (WLF) Equation [26] predicts the appropriate temperature dependence of the horizontal shift factors related through the constants $c_{1}$ and $c_{2}$ by the relation

$\log a_{T}=\frac{-c_{1}\left(T-T_{r e f}\right)}{c_{2}+\left(T-T_{\text {ref }}\right)}$

Fig. 8 shows the master curve obtained from the tTS superposition of the viscoelastic response data in the homogenous region of the 50:50 PSD-500 K/PVME-90 K blend. The reference temperature here is $150^{\circ} \mathrm{C}$, well below the SANSdetermined critical temperature $\left(\sim 173^{\circ} \mathrm{C}\right)$ of the blend. In the lower frequency region, scaling behaviours of $G^{\prime}-\omega^{2}$ and $G^{\prime \prime}-\omega^{1}$ are clearly observed throughout the temperature range of the experiment as evident from the near perfect superposition of the data in the master curve, a characteristic typically associated with the behaviour of blends in the terminal region. The values of the fitting parameters $c_{1}$ and $c_{2}$ obtained from a linear regression fit of the modulus-scale shift factor data to Eq. (2), at a reference temperature of $150{ }^{\circ} \mathrm{C}$ are 4.5 and 307.8 , respectively, for the (master curve) data shown in Fig. 8. A similar master curve has also been obtained for yet another blend of 50:50 PSD-300 K/ PVME-90 K by shifting the isothermal frequency-sweep data obtained in the homogenous region on to the data at a reference temperature of $110{ }^{\circ} \mathrm{C}$ (not shown here for the sake of brevity). The values of the fitting parameters in case of PSD-300 K/ PVME-90 K blend were found to be 2.2 and 125.4 , respectively. Fits of the modulus-scale shift factor data plotted on inverse temperature scale for 50:50 blends of PSD-300 K/PVME-90 K and

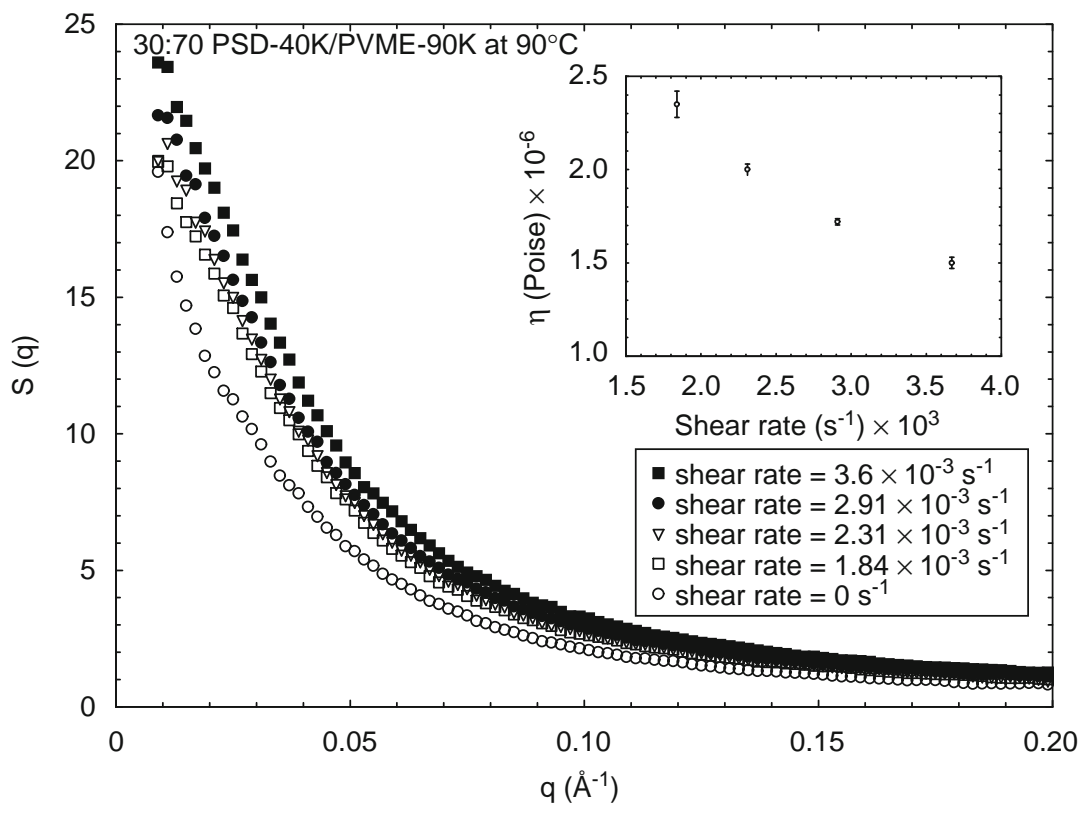

Fig. 7. SANS profile of 30:70 PSD-40 K/PVME-90 K blend obtained at a fixed temperature of $90{ }^{\circ} \mathrm{C}$ at both the zero shear (quiescent) and varying steady shear conditions. The inset shows the corresponding variation in the viscosity. 


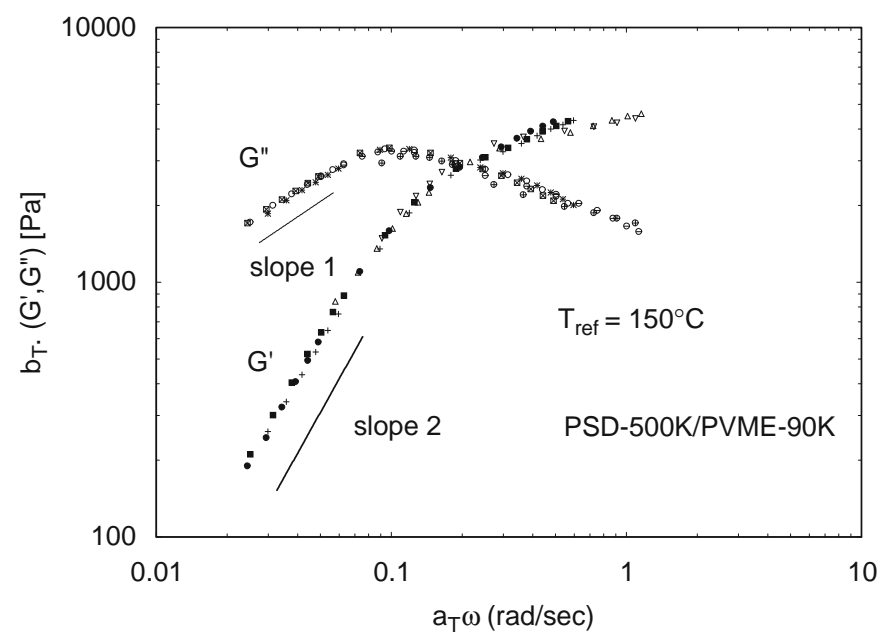

Fig. 8. Master curves of storage $\left(G^{\prime}\right)$ and loss modulus $\left(G^{\prime \prime}\right)$ for a $50: 50500 \mathrm{~K} / 90 \mathrm{~K}$ PSD/PVME blend, as a function of frequency, $\omega$ at a reference temperature of $150{ }^{\circ} \mathrm{C}$, where $a_{T}$ and $b_{T}$ are the frequency-scale and modulus-scale shift factors, respectively. Scaling behaviour of $G^{\prime}-\omega^{2}$ and $G^{\prime \prime}-\omega^{1}$ at lower frequencies (in the terminal region) is shown in the figure.

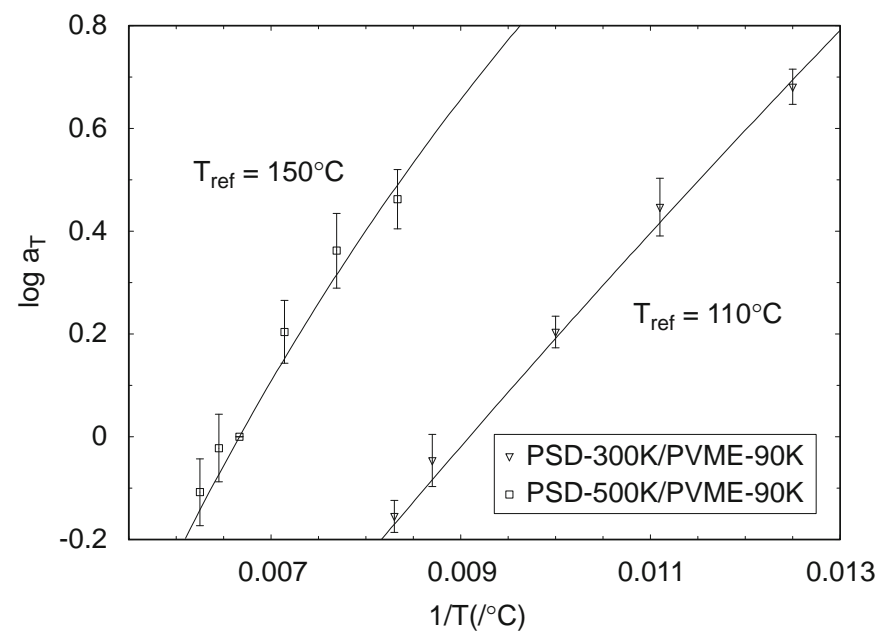

Fig. 9. Temperature dependence of the frequency-scale shift factor, $a_{T}$ (on the logarithmic scale) for the master curves obtained at reference temperatures of 110 and $150{ }^{\circ} \mathrm{C}$ for the blends of PSD-300 K/PVME-90 $\mathrm{K}$ and PSD-500/PVME-90 K, respectively. The values of the fitting parameters $c_{1}$ and $c_{2}$ obtained from the regression fits to the data for the former and the latter are 2.2 and 125.4, and 4.5 and 307.8 , respectively.

PSD-500 K/PVME-90 $\mathrm{K}$ are shown in Fig. 9. The values of the fitting parameters obtained for both the blends are found to be consistent with those obtained by other authors [30] for the blends of polystyrene (PS) and PVME.

Another important aspect of study involving blends of $\mathrm{PS} / \mathrm{PVME}$ is concerned with the observation of the tTS breakdown near the phase boundary. Rheology and dielectric relaxation experiments performed on the blends of PS/PVME suggest [25] the empirical time-temperature superposition (tTS) principle breaks down in the homogenous region but close to the critical region, manifesting a thermo-rheologically complex behaviour. Since our studies on the blends of PSD and PVME are almost all confined in a region far from the critical temperature, the possibility for such an observation does not arise while the parameters obtained here essentially obeys all the characteristics of the viscoelastic response behaviour expected for the blends of $\mathrm{PS} / \mathrm{PVME}$ in the homogenous region. Experiments near the critical region and further discussion on the aspects of tTS breakdown are beyond the scope of this paper and will be dealt with in our future studies.

\section{Conclusions and future work}

In summary, the apparatus described here features unique capabilities for simultaneous rheology and in situ SANS measurements compared to other available instruments. While other parallel plate shear devices for in situ measurements are either not capable to perform steady shear (restricted to oscillatory mode only) [20] or are not equipped with appropriate strain sensors for rheological measurements [3], our apparatus is capable of performing both the operations. A major advantage of using such apparatus over the Couette type cells is its ability to perform simultaneous measurements on high-viscosity samples for which the latter is not suitable and minimization of the possibility of multiple scattering through a reduction in the path length of the beam traversed for the same thickness of the sample. Experiments performed on the high-viscosity polymeric blends of PSD/PVME clearly demonstrate the unique capabilities and salient features of the apparatus.

Despite the state-of-art current design and existing operational features of apparatus it can be improved on several counts to enable more efficient measurements in future. For example, plates can be designed to hold samples only in a small annular region instead of spreading them all over the plate, which will significantly reduce the volume of sample required to perform such experiments along with an added cost benefit resulting from the minimization of deuterated sample requirements. Introducing a geared motor will allow more sensitive measurements to be performed across a wide range of shear rates and sampleviscosities.

\section{Acknowledgements}

Financial support for the research is gratefully acknowledged from the Engineering and Physical Sciences Research Council. The Council for the Central Laboratories of the Research Councils and the ISIS Facility are acknowledged for the provision of neutron beam time. We are also grateful to Lian Hutchings and the technical staffs of both Durham University and the Rutherford Appleton Laboratory for synthesizing the deuterated polystyrene used in the work and adapting the clamshell oven. We also express our gratitude to Richard Thompson for his assistance during experiments at RAL.

\section{References}

[1] C. Munch, J. Kalus, Rev. Sci. Instrum. 70 (1999) 187.

[2] K. Matsuzaka, T. Hashimoto, Rev. Sci. Instrum. 70 (1999) 2387.

[3] J. Kalus, G. Neubauer, U. Schmeizer, Rev. Sci. Instrum. 61 (1990) 3384.

[4] J. Sharma, N. Clarke, J. Phys. Chem. B 108 (2004) 13220.

[5] B.J. Ackerson, N.A. Clark, Phys. Rev. A 30 (1984) 906.

[6] P. Lindner, R.C. Oberthur, Coll. Poly. Sci. 266 (1988) 886.

[7] I.W. Hamley, J.A. Pople, C. Booth, L. Derici, M. Impéror-Clerc, P. Davidson, Phys. Rev. E 58 (1998) 7620.

[8] V.M. Cloke, J.S. Higgins, C.L. Phoon, S.M. Richardson, S.M. King, R. Done T.E. Cooper, Rev. Sci. Instrum. 67 (1996) 3158.

[9] E. Cappelaere, J.F. Berret, J.P. Decruppe, P. Lindner, Phys. Rev. E 56 (1997) 1869.

[10] J. Zipfel, J. Berghausen, P. Lindner, P. Richtering, J. Phys. Chem. B 103 (1999) 2841.

[11] I.A. Hindawi, J.S. Higgins, R.A. Weiss, Polymer 33 (1992) 2522.

[12] S. Mani, M.F. Malone, H.H. Winter, Macromolecules 25 (1992) 5671.

[13] K. Krishnan, K. Almdal, W.R. Burghardt, T.P. Lodge, F.S. Bates, Phys. Rev. Lett. 87 (2001) 098301.

[14] J.B. Hayter, J. Penfold, J. Phys. Chem. 88 (1984) 4589. 
[15] S.M. King, R.K. Heenan, V.M. Cloke, C. Washington, Macromolecules 30 (1997) 6215.

[16] J. Penfold, E.J. Staples, A.K. Lodi, I. Tucker, G.J.T. Tiddy, J. Phys. Chem. B 101 (1997) 66.

[17] N.P. Balsara, B. Hammouda, P.K. Kesani, S.V. Jonnalagadda, G.C. Straty, Macromolecules 27 (1994) 2566.

[18] J.-F. Berret, D.C. Roux, G. Porte, P. Lindner, Europhys. Lett. 25 (1994) 521

[19] F. Boue, P. Lindner, Europhys. Lett. 25 (1994) 421.

[20] K. Mortensen, A. Almdal, R. Kleppinger, N. Mischenko, H. Reynaers, Physica B 241 (1997) 1025.

[21] Y. Matsushita, H. Furuhashi, H. Choshi, I. Noda, M. Nagasawa, T. Fujimoto C.C. Han, Polym. J. 14 (1982) 489.

[22] 〈http://www.isis.stfc.ac.uk/instruments/loq/loq2470.html 〉.
[23] http://www.isis.stfc.ac.uk/.

[24] S.M. King, R.K. Heenan, Using COLETTE, Rutherford Appleton Laboratory Report RAL-95-005 1995.

[25] J.A. Pathak, R.H. Colby, G. Floudas, R. Jerome, Macromolecules 32 (1999) 2553.

[26] J.D. Ferry, in: Viscoelastic Properties of Polymers, 3rd ed., Wiley, New York, 1980.

[27] D. Schwahn, K. Mortensen, H. Yee-Madeira, Phys. Rev. Lett. 58 (1987) 1544

[28] T. Nishi, T.T. Wang, T.K. Kwei, Macromolecules 8 (1975) 227.

[29] P.G. de Gennes, in: Scaling Concepts in Polymer Physics, Cornell University Press, Ithaca, New York, 1979.

[30] M. Kapnistos, A. Hinrichs, D. Vlassopoulos, S.H. Anastasiadis, A. Stammer B.A. Wolf, Macromolecules 29 (1996) 7155. 